\title{
Best Technology Assessment Areas Linear Array on the Source when Exposed to Corre-lated Noise with Unknown Functions Distribution
}

\author{
Sergei V. Shostak, \\ Paul A. Starodubtsev* and Roman N. Aliafanov \\ Far Eastern Federal University \\ 8 Sukhanova, Vladivostok, 690950, Russia
}

Received 12.01.2017, received in revised form 10.02.2017, accepted 28.02.2017

\begin{abstract}
We obtain an unbiased estimate method for direction to the source of radiation with minimum variance in conditions of a linear array antenna correlation interference from an unknown distribution function. Meeting the challenge of minimizing the variance and to obtain unbiased estimates are based on the Gauss-Markov theorem. The model of the formation of a discrete space-time signal in the form of a generalized vector. Based on analysis of Cramer-Rao inequality rating direction is produced by the harmonic expansion in the spatial coordinate, which allows for assessment without the formation of the traditional directional characteristics. Formed and presented the implementation of an algorithm derived method.
\end{abstract}

Keywords: multi-element antenna arrayб, spatiotemporal signal, echo, antenna aperture, correlation, periodogram.

Citation: Shostak S.V., Starodubtsev P.A., Aliafanov R.N. Best technology assessment areas linear array on the source when exposed to correlated noise with unknown functions distribution, J. Sib. Fed. Univ. Eng. technol., 2017, 10(2), $249-259$. DOI: $10.17516 / 1999-494 X-2017-10-2-249-259$.

(C) Siberian Federal University. All rights reserved

* Corresponding author E-mail address: spa1958@mail.ru,gidra_518@mail.ru 


\title{
Технология оптимальной оценки
}

направления линейной антенной решетки

на источник сигнала при воздействии

на нее коррелированных помех

с неизвестной функцией распределения

\author{
С.В. Шостак, П.А. Стародубцев, Р.Н. Алифанов \\ Дальневосточный федеральный университет \\ Россия, 690950, Владивосток, Суханова, 8
}

\begin{abstract}
Получен метод несмещенной оценки направления на источник излучения с минимальной дисперсией в условиях воздействия на линейную антенную решетку корреляционных помех с неизвестной функиией распределения. Решение задачи минимизации дисперсии и получения несмещенной оценки базируется на теореме Гаусса-Маркова. Представлена модель формирования дискретного пространственно-временного сигнала в виде обобщенного вектора. На основе анализа неравенства Рао-Крамера оченка направления производится путем гармонического разложения по пространственной координате, что позволяет проводить оиенку без формирования традиционной характеристики направленности. Сформирован и представлен алгоритм реализации полученного метода.
\end{abstract}

Ключевые слова: многоэлементная антенная решетка, пространственно-временной сигнал, эхо-сигнал, апертура антенны, коррелячия, периодограмма.

\section{Введение}

При разработке современных гидроакустических систем дальнего обнаружения морских объектов и физических явлений значительное внимание уделяется методам обработки сигналов в эквидистантных линейных антенных решетках. Они представляют собой сенсорные системы, состоящие из размещенных на одинаковом расстоянии гидрофонов, предназначенных для интерпретации акустических волн. Обработку сигналов в указанных эквидистантных линейных решетках необходимо понимать как задачу оптимальной многоканальной фильтрации, основной целью которой является не только обнаружение полезного сигнала, но и оценка его параметров, несущих информацию о пространственном положении источника колебаний. При этом распространение сигнала происходит при наличии шума окружающей среды.

В связи с тем что многоэлементная антенная решетка в задачах гидролокации используется в основном для пространственной фильтрации или угловой селекции, многие работы по этим вопросам посвящены в основном методам получения требуемых диаграмм направленности с помощью весового суммирования сигналов отдельных элементов решетки. В основе такого подхода лежит предположение, что наилучшим способом, обеспечивающим большую эффективность обнаружения сигнала, является формирование некоторой диаграммы направленности решетки. Так как волна по определению есть функция пространства и времени, т.е. имеется функциональная зависимость между пространственной и временной переменными, то при более общем подходе к проблеме обнаружения сигнала и определения угловых характеристик его источника на форму диаграммы направленности не накладывается каких-либо

$$
-250-
$$


ограничений. В этом случае технологии волновой обработки, включающие пространственное и временное измерения и основанные на методах пространственно-временной обработки сигналов, позволяют получить более значительное увеличение эффективности, чем при обычном подходе.

\section{Основная часть}

Обработка пространственно-временного сигнала обычно проводится в присутствии в гидроакустическом канале шумов и помех среды. Поэтому для таких условий надо говорить об оценке направления, т.е задача определения направления на источник уже относится к области статистической обработки. В таком случае для решения поставленной задачи необходимо предварительно задать статистические характеристики шума, определить критерии оптимальности и модель формирования пространственно-временного сигнала.

В традиционных методах оценки направления, как правило, предполагается, что функция распределения шума известна, шумы на гидрофонах не коррелированны и имеют одинаковую дисперсию. В реальной обстановке такие предположения выполняются редко. Например, помеха от точечного отражателя/излучателя формирует на решетке коррелированную помеху, в результате чего в гидрофонах помехи не только становятся коррелированными, но и имеют различную дисперсию [1]. В данной работе будем считать, что на интервале наблюдения шум (сюда входят и помехи) является стационарным процессом с нулевым средним, неизвестной функцией распределения, не обязательно белый. В качестве критерия оптимальности примем, что оценка должна быть несмещенной и с минимальной дисперсией.

Рассмотрим теперь модель пространственно-временного сигнала, формируемого линейной антенной решеткой.

В предположении плоского волнового фронта поле акустического давления формирует пространственно-временной сигнал, как показано на рис. 1.

Полагаем, что цель излучает/отражает гармонический сигнал вида

$$
s(t)=a \cdot \cos \left(2 \pi f_{0}^{\prime} t-\Psi\right),
$$

где $\alpha$ - амплитуда сигнала; $f_{0}^{\prime}$ - несущая частота; $\Psi$ - начальная фаза.

Считаем, что антенная решетка расположена в дальней зоне, когда волновой фронт сигнала плоский, как представлено на рис. 1. В таком случае сигнал на $m$-м гидрофоне запаздывает относительно ( $m-1)$-го на величину $\frac{d}{c} \sin \varphi_{0}$, где $d$ - расстояние между гидрофонами, $c$ - скорость звука в воде. Тогда на отдельном гидрофоне формируется информационный сигнал

$$
\begin{aligned}
& s(m, t)=a \cdot \cos \left(2 \pi f_{0}^{\prime}\left(t-\frac{d}{c} \sin \varphi_{0} \cdot m\right)-\Psi\right)= \\
& =a \cdot \cos \left(2 \pi f_{0}^{\prime} t-2 \pi\left(\frac{d}{\lambda_{0}} \sin \varphi_{0}\right) m-\Psi\right),
\end{aligned}
$$

где $\lambda_{0}-$ длина волны. 


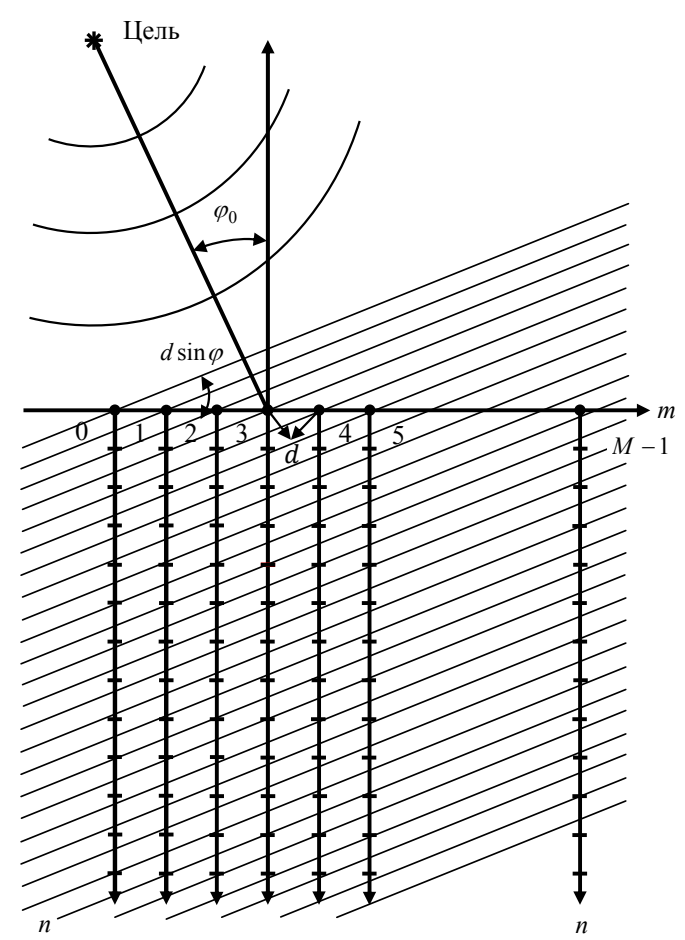

Рис. 1. Модель формирования пространственно-временного сигнала

После аналого-цифрового преобразования сигналов в каждом канале выражение (2) становится

$$
s(m, n)=a \cdot \cos \left(2 \pi f_{0} n-2 \pi\left(\frac{d}{\lambda_{0}} \sin \varphi_{0}\right) m-\Psi\right),
$$

где $n=0 \div N-1-$ номер отсчет в области времени; $f_{0}=f_{0}^{\prime} / f_{\text {д }} ; f_{д}$ - частота дискретизации.

В реальной обстановке информационный сигнал (3) приходит на антенную решетку в аддитивной смеси с шумом гидроакустического канала $\omega(m, n)$.

В результате на выходе гидрофона формируется сигнал

$$
x(m, n)=s(m, n)+\omega(m, n),
$$

где $\omega(m, n)$ - шумовая составляющая, которая включает и помехи.

Перепишем выражение (4) в развернутом виде:

$$
\begin{aligned}
& x(m, n)=a \cdot \cos \left(2 \pi f_{0} n-2 \pi\left(\frac{d}{\lambda_{0}} \sin \varphi_{0}\right) m-\Psi\right)+\omega(m, n)= \\
& =a \cdot \cos \left(2 \pi f_{0} n\right) \cdot \cos \left(2 \pi \vartheta_{0} m-\Psi\right)+a \cdot \sin \left(2 \pi f_{0} n\right) \cdot \sin \left(2 \pi \vartheta_{0} m-\Psi\right)+\omega(m, n),
\end{aligned}
$$

где $\vartheta_{0}=\frac{d}{\lambda_{0}} \sin \varphi_{0}$. 
Раскрывая изменение сигнала во времени (по переменной $n$ ), получим

$$
\begin{aligned}
& x(m, 0)=a \cdot \cos \left(2 \pi f_{0} \cdot 0\right) \cdot \cos \left(2 \pi \vartheta_{0} m-\Psi\right)+a \cdot \sin \left(2 \pi f_{0} \cdot 0\right) \cdot \sin \left(2 \pi \vartheta_{0} m-\Psi\right)+\omega(m, 0) \\
& x(m, 1)=a \cdot \cos \left(2 \pi f_{0} \cdot 1\right) \cdot \cos \left(2 \pi \vartheta_{0} m-\Psi\right)+a \cdot \sin \left(2 \pi f_{0} \cdot 1\right) \cdot \sin \left(2 \pi \vartheta_{0} m-\Psi\right)+\omega(m, 1) \\
& x(m, N-1)=a \cdot \cos \left(2 \pi f_{0} \cdot(N-1)\right) \cdot \cos \left(2 \pi \vartheta_{0} m-\Psi\right)+ \\
& +a \cdot \sin \left(2 \pi f_{0} \cdot(N-1)\right) \cdot \sin \left(2 \pi \vartheta_{0} m-\Psi\right)+\omega(m, N-1)
\end{aligned}
$$

Выражение (6) несложно представить в векторно-матричной форме

$$
\mathbf{X}_{\mathbf{m}}=\mathbf{H}^{\prime} \cdot \boldsymbol{\theta}_{\mathbf{m}}^{\prime}+\mathbf{W}_{\mathbf{m}}
$$

где

$$
\begin{gathered}
\mathbf{X}_{\mathbf{m}}=\left[\begin{array}{c}
x(m, 0) \\
x(m, 1) \\
\vdots \\
x(m, N-1)
\end{array}\right] ; \quad \mathbf{H}^{\prime}=\left[\begin{array}{cc}
1 & 0 \\
\cos \left(2 \pi f_{0} \cdot 1\right) & \sin \left(2 \pi f_{0} \cdot 1\right) \\
\vdots & \vdots \\
\cos \left(2 \pi f_{0}(N-1)\right) & \sin \left(2 \pi f_{0}(N-1)\right)
\end{array}\right] ; \\
\mathbf{\theta}_{\mathbf{m}}^{\prime}=\left[\begin{array}{c}
a \cdot \cos \left(2 \pi \vartheta_{0} m-\Psi\right) \\
a \cdot \sin \left(2 \pi \vartheta_{0} m-\Psi\right)
\end{array}\right]=a\left[\begin{array}{c}
\omega(m, 0) \\
\cos \left(2 \pi \vartheta_{0} m-\Psi\right) \\
\sin \left(2 \pi \vartheta_{0} m-\Psi\right)
\end{array}\right] ; \quad \mathbf{W}_{\mathbf{m}}=\left[\begin{array}{c}
\omega(m, 1) \\
\vdots \\
\omega(m, N-1)
\end{array}\right] ;
\end{gathered}
$$

из соотношения (7) следует, что информацию об амплитуде сигнала $a$ и направлении на источник $\varphi_{0}$ несет вектор $\boldsymbol{\theta}_{\mathbf{m}}^{\prime}$ при искажающем воздействии шумового вектора $\mathbf{W}_{\mathbf{m}}$. Для получения последующих результатов представим пространственно-временной сигнал, сформированный антенной решеткой, в общем векторно-матричном виде, в результате чего образуются следующие выражения:

$$
\mathbf{X}=\left[\begin{array}{c}
\mathbf{X}_{\mathbf{0}} \\
\vdots \\
\mathbf{X}_{\mathbf{M}-\mathbf{1}}
\end{array}\right]=\underbrace{\left[\begin{array}{c}
x(0,0) \\
\cdots \ldots \\
x(0, N-1) \\
x(1,0) \\
\ldots \ldots \\
x(1, N-1) \\
\cdots \cdots \\
x(M-1,0) \\
\cdots \ldots \\
x(M-1, N-1)
\end{array}\right]}_{M N \times 1}
$$

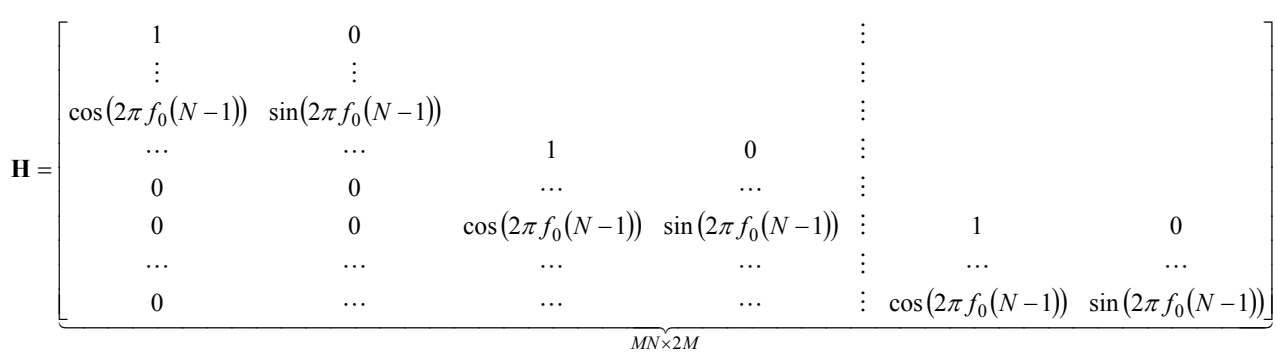




$$
\begin{gathered}
\boldsymbol{\theta}=\left[\begin{array}{c}
\boldsymbol{\theta}_{\mathbf{0}}^{\prime} \\
\vdots \\
\boldsymbol{\theta}_{\mathbf{M}-1}^{\prime}
\end{array}\right]=\underbrace{\left[\begin{array}{c}
\cos \left(2 \pi \vartheta_{0} \cdot 0-\Psi\right) \\
\sin \left(2 \pi \vartheta_{0} \cdot 0-\Psi\right) \\
\vdots \\
\cos \left(2 \pi \vartheta_{0} \cdot(M-1)-\Psi\right) \\
\sin \left(2 \pi \vartheta_{0} \cdot(M-1)-\Psi\right)
\end{array}\right]}_{2 M \times 1} \\
\mathbf{W}=\left[\begin{array}{c}
\mathbf{W}_{\mathbf{0}} \\
\vdots \\
\mathbf{W}_{\mathbf{M}-1}
\end{array}\right]=\underbrace{\left[\begin{array}{c}
\omega(0,0) \\
\ldots \ldots \\
\omega(0, N-1) \\
\ldots \ldots \\
\omega(M-1,0) \\
\ldots \ldots \\
\omega(M-1, N-1)
\end{array}\right]}_{M N \times 1} .
\end{gathered}
$$

Окончательно в компактном виде модель пространственно-временного сигнала решетки можно записать таким образом:

$$
\mathbf{X}=\mathbf{H} \cdot \boldsymbol{\theta}+\mathbf{W},
$$

где $\mathbf{X}$ - вектор измерений размерностью $(M N \times 1)$; $\mathbf{H}$ - матрица связи размерностью $(M N \times 2 M)$; $\boldsymbol{\theta}$ - вектор параметров размерностью $(2 M \times 1) ; \mathbf{W}-$ вектор шума размерностью $(M N \times 1)$.

В выражении (12) вектор $\mathbf{X}$ является вектором сигналов, измеренных на гидрофонах, $\mathbf{H}-$ известная матрица.

Из выражения (12) видно, что модель формирования сигнала в антенной решетке представляется в виде линейного векторно-матричного уравнения, где искомой величиной выступает вектор $\boldsymbol{\theta}$. Для оценки $\boldsymbol{\theta}$ в подобного рода моделях применимы методы линейного оценивания, когда оценки вычисляются как линейные комбинации взвешенных с определенными весами, которые необходимо определить, наблюдаемых сигналов [2, 3]. К достоинствам таких оценок относится возможность получать несмещенные оценки, а также то, что они не требуют точного знания статистик шума, а только моменты до второго порядка включительно.

Обобщенный метод линейного оценивания основан на выражении

$$
\hat{\boldsymbol{\theta}}=\mathbf{A} \cdot \mathbf{X},
$$

где $\mathbf{A}$ - неизвестная матрица весов, которую требуется определить.

Как известно, основным требованием к методам оценивания является требование выделения насколько можно точнее некоторых величин из зашумленных данных $[2,3,4]$. К такому требованию относится требование несмещенности оценки

$$
\mathbf{E}[\hat{\boldsymbol{\theta}}]=\boldsymbol{\theta},
$$

где $\mathbf{E}$ - оператор математического ожидания.

Из-за наличия шума при оценке $\hat{\boldsymbol{\theta}}$ снова формируется случайный процесс, и тогда метод линейного оценивания дает 


$$
\mathbf{E} \mid \hat{\boldsymbol{\theta}}]=\mathbf{E}[\mathbf{A X}]=\mathbf{E}[\mathbf{A}(\mathbf{H} \boldsymbol{\theta}+\mathbf{W})]=\mathbf{E}[\mathbf{A H \theta} \cdot \mathbf{A W}]=\mathbf{A H E}[\boldsymbol{\theta}]+\mathbf{A E}[\mathbf{W}]=\mathbf{A H \theta}+\mathbf{A E}[\mathbf{W}]
$$

Так как ранее было положено, что среднее значение шума равно нулю, т.е. $\mathbf{E}[\mathbf{W}]=\mathbf{0}$, то матрица А должна удовлетворять условию

$$
\mathbf{A} \cdot \mathbf{H}=\mathbf{I},
$$

где I - единичная матрица.

Получим теперь выражение для дисперсии вектора оценки $\hat{\boldsymbol{\theta}}$ через ковариационную матрицу

$$
\mathbf{E}\left[(\hat{\boldsymbol{\theta}}-\boldsymbol{\theta})(\hat{\boldsymbol{\theta}}-\boldsymbol{\theta})^{\mathrm{T}}\right]=\mathbf{E}\left[(\mathbf{A X}-\boldsymbol{\theta})(\mathbf{A X}-\boldsymbol{\theta})^{\mathrm{T}}\right]=\mathbf{E}\left[(\mathbf{A H} \boldsymbol{\theta}+\mathbf{A W}-\boldsymbol{\theta})(\mathbf{A H} \boldsymbol{\theta}-\mathbf{A W}-\boldsymbol{\theta})^{\mathrm{T}}\right]
$$

где Т - операция транспортирования.

С учетом условий (16) получим

$$
\mathbf{E}\left[(\hat{\boldsymbol{\theta}}-\boldsymbol{\theta})(\hat{\boldsymbol{\theta}}-\boldsymbol{\theta})^{\mathrm{T}}\right]=\mathbf{E}\left[(\mathbf{A W})(\mathbf{A W})^{\mathrm{T}}\right]=\mathbf{E}\left[\mathbf{A} \mathbf{W} \mathbf{W}^{\mathrm{T}} \mathbf{A}^{\mathrm{T}}\right]=\mathbf{A E}\left[\mathbf{W} \mathbf{W}^{\mathrm{T}}\right] \mathbf{A}^{\mathrm{T}}=\mathbf{A} \mathbf{C}_{\mathbf{W}} \mathbf{A}^{\mathrm{T}}
$$

где $\mathbf{C}_{\mathbf{W}}$ - ковариационная матрица шума.

Кроме требования несмещенности, другим требованием к оценке служит оценивание с минимальной дисперсией. Для этого необходимо найти матрицу весовых коэффициентов $\mathbf{A}$, которая давала бы минимальное значение (17) с учетом условий (16). Эта задача на поиск условного минимума и решение ее получено в [2-4] на основе теоремы Гаусса-Маркова, из которой следует, что если данные измерений есть общая линейная модель вида

$$
\mathbf{X}=\mathbf{H} \cdot \boldsymbol{\theta}+\mathbf{W},
$$

где $\mathbf{H}$ - известная $(M N \times 2 M)$ матрица, $\boldsymbol{\theta}$ есть $(2 M \times 1)$ вектор параметров для оценивания, $\mathbf{W}$ есть $(M N \times 1)$ произвольно распределенный шумовой вектор с нулевым средним и известной $(M N \times M N)$ ковариационной матрицей $\mathbf{C}_{\mathbf{w}}$, то наилучшей линейной несмещенной оценкой для $\hat{\boldsymbol{\theta}}$ является

$$
\hat{\boldsymbol{\theta}}=\left(\mathbf{H}^{\mathrm{T}} \mathbf{C}_{\mathbf{W}} \mathbf{H}\right)^{-1} \mathbf{H}^{\mathrm{T}} \mathbf{C}_{\mathbf{W}}^{-1} \mathbf{X}
$$

При этом дисперсия оценки $\hat{\boldsymbol{\theta}}$ определяется выражением

$$
\operatorname{var}(\hat{\boldsymbol{\theta}})=\left(\mathbf{H}^{\mathrm{T}} \mathbf{C}_{\mathbf{W}}^{-1} \mathbf{H}\right)^{-1},
$$

а минимальная дисперсия оценки отдельного параметра $\hat{\theta}_{m}$

$$
\operatorname{var}\left(\hat{\theta}_{m}\right)=\left[\left(\mathbf{H}^{T} \mathbf{C}^{-1} \mathbf{H}\right)^{-1}\right]_{m m} .
$$

Для нашего случая получили, что

$$
\mathbf{A}=\left(\mathbf{H}^{\mathrm{T}} \mathbf{C}_{\mathbf{W}}^{-1} \mathbf{H}\right)^{-1} \mathbf{H}^{\mathrm{T}} \mathbf{C}_{\mathbf{W}}^{-1} .
$$

В частном случае, если $\mathbf{W}$ белый шум с ковариационной матрицей 


$$
\mathbf{C}_{\mathbf{W}}=\sigma_{W}^{2} \cdot \mathbf{I}
$$

где $\sigma_{W}^{2}$ - дисперсия шума, то $\mathbf{C}_{\mathbf{W}}^{\mathbf{- 1}}=\frac{1}{\sigma_{W}^{2}} \cdot \mathbf{I}$, и тогда выражение оценки (19) приобретает вид

$$
\hat{\boldsymbol{\theta}}=\left(\mathbf{H}^{\mathrm{T}} \mathbf{C}^{-1} \mathbf{H}\right)^{-1} \cdot \mathbf{H}^{\mathrm{T}} \mathbf{C}^{-1} \mathbf{X}=\left(\mathbf{H}^{\mathrm{T}} \frac{1}{\sigma_{\mathrm{W}}^{2}} \mathbf{I} \cdot \mathbf{H}\right)^{-1} \cdot \mathbf{H}^{\mathrm{T}}\left(\frac{1}{\sigma_{\mathrm{W}}^{2}} \mathbf{I}\right)^{-1} \cdot \mathbf{X}=\left(\mathbf{H}^{\mathrm{T}} \mathbf{H}\right)^{-1} \cdot \mathbf{H}^{\mathrm{T}} \mathbf{X},
$$

т.е. сводится к стандартной процедуре наименьших квадратов.

Множитель $\mathbf{C}_{\mathbf{W}}^{-1}$ в (19) проводит предварительное предотбеливание данных до усреднения, т.е. выравнивает шумовой вклад каждого гидрофона. Тогда, если $\mathbf{W}$ имеет гауссовское распределение, то в результате вектор $\hat{\boldsymbol{\theta}}$ также будет распределен по нормальному закону с ковариационной матрицей вида (22).

Как показывает (10), вектор $\hat{\boldsymbol{\theta}}$ состоит из ряда косинусов и синусов, искаженных шумом, который приведен к белому. Последовательности косинусов и синусов в $\hat{\boldsymbol{\theta}}$ являются функциями, где в качестве аргумента выступает номер гидрофона, а постоянная величина $\vartheta_{0}=\frac{d}{\lambda_{0}} \cdot \sin \varphi_{0}$ имеет смысл пространственной частоты, т.е.

$$
\begin{aligned}
& \cos \left(2 \pi \vartheta_{0} m-\Psi\right)=\cos \left(2 \pi\left(\frac{d}{\lambda_{0}} \sin \varphi_{0}\right) m-\Psi\right) \\
& \sin \left(2 \pi \vartheta_{0} m-\Psi\right)=\sin \left(2 \pi\left(\frac{d}{\lambda_{0}} \sin \varphi_{0}\right) m-\Psi\right) .
\end{aligned}
$$

В результате задача оценки направления на источник сводится к оценке пространственной частоты $\square_{0}$ гармонического колебания на фоне белого шума с минимальной дисперси ей.

Вектор оценок параметров (19) с помощью $(M \times 2 M)$ матрицы

$$
\mathbf{D}=\underbrace{\left[\begin{array}{llllll}
1 & j & 0 & 0 & \cdots & 0 \\
0 & 0 & 1 & j & \cdots & 0 \\
\vdots & \vdots & \vdots & \vdots & \vdots & \vdots \\
0 & \cdots & \cdots & \cdots & 1 & j
\end{array}\right],}_{M \times 2 M}
$$

где $j=\sqrt{-1}-$ мнимая единица, несложно преобразовать в комплексный вид

$$
\mathbf{Z}=\mathbf{D} \cdot \hat{\boldsymbol{\theta}}
$$

Далее вектор $\mathbf{Z}$ легко представить в виде комплексного сигнала переменной $m$ :

$$
z(m)=a \cdot \exp \left[j\left(2 \pi\left(\frac{d}{\lambda_{0}} \sin \varphi_{0}\right) m-\Psi\right)\right]+\varepsilon(m)=a \cdot \exp \left[j\left(2 \pi \vartheta_{0} m-\Psi\right)\right]+\varepsilon(m)
$$

где $\varepsilon(m)$ - шумовая составляющая с дисперсией $\sigma_{\varepsilon}^{2}$ и ковариационной матрицей $\sigma_{\varepsilon}^{2} \cdot \mathbf{I}$. 
В работе [5] проведено решение задачи оценки амплитуды, частоты и начальной фазы гармонического сигнала вида (28) на фоне белого гауссовского шума методом максимального правдоподобия. Представленные результаты показывают, что наилучшим методом оценки частоты является метод периодограммы $[5,6]$, который для нашего случая имеет вид

$$
\frac{1}{M}\left|\sum_{m=0}^{M-1} z(m) \cdot \exp (-j 2 \pi \vartheta \cdot m)\right|^{2},
$$

где | | - абсолютное значение (модуль) выражения.

Подставим в (29) информационную часть (28):

$$
\begin{aligned}
& \frac{1}{M}\left|\sum_{m=0}^{M-1} a \cdot \exp \left[j\left(2 \pi \vartheta_{0} m-\Psi\right) \cdot \exp (-j 2 \pi \vartheta m)\right]\right|^{2}= \\
& =\frac{1}{M}\left|a \sum_{m=0}^{M-1} \exp \left[-j 2 \pi\left(\vartheta-\vartheta_{0}\right) \cdot m\right] \cdot \exp (-j \Psi)\right|^{2}= \\
& =\frac{a^{2}}{M}\left|\sum_{m=0}^{M-1} \exp \left[-j 2 \pi\left(\vartheta-\frac{d}{\lambda_{0}} \sin \varphi_{0}\right) \cdot m\right]\right|^{2} .
\end{aligned}
$$

Несложно заметить, что в случае $\vartheta=\vartheta_{0}=\frac{d}{\lambda_{0}} \cdot \sin \varphi_{0}$ (30) имеет максимальное значение.

Следовательно, оценка пространственной частоты $\hat{\vartheta}_{0}$ и угла $\square_{0}$ соответственно определяется выбором частоты $\square$, для которой периодограмма достигает максимального значения. При этом неравенство Рао-Крамера дисперсии оценки $\hat{\vartheta}_{0}$ имеет вид $[2,5]$

$$
\operatorname{var}\left(\hat{\vartheta}_{0}\right) \geq \frac{6 \sigma_{\varepsilon}^{2}}{a^{2} \cdot M\left(M^{2}-1\right)(2 \pi)^{2}}=\frac{6}{\left(\frac{a^{2}}{\sigma_{\varepsilon}^{2}}\right) M\left(M^{2}-1\right)}=\frac{6}{q M\left(M^{2}-1\right)},
$$

где $q=\frac{a^{2}}{\sigma_{\varepsilon}^{2}}-$ отношение сигнал/шум.

Выражение (31) показывает, что граница Рао-Крамера оценки пространственной частоты обратно пропорциональна отношению сигнал/шум и уменьшается обратно пропорционально третьей степени числа гидрофонов в линейной антенной решетке, что делает рассмотренный способ достаточно помехоустойчивым.

По предложенной технологии оценки направления на источник излучения несложно сформулировать алгоритм обработки сигналов, который содержит совокупность следующих методов и инструментов:

1) оценка автокорреляционной матрицы помех $\mathbf{C}_{\mathbf{w}}$;

2) формирование вектора входных сигналов в соответствии с (8);

3) формирование матрицы Н в соответствии с (9);

4) получение матрицы А в соответствии с (22);

5) вычисление $\hat{\boldsymbol{\theta}}$ в соответствии с (13);

6) формирование матрица $\mathbf{D}$ в соответствии с (26); 
7) определение $\mathbf{Z}$ в соответствии с (27);

8) вычисление периодограммы (29);

9) нормирование частотной оси периодограммы в угловых единицах.

\section{Заключение}

Таким образом, в настоящей работе получена технология оптимальной оценки направления на источник сигнала для линейной антенной решетки при воздействии на нее некоррелированных помех с неизвестной функцией распределения. При этом предполагается, что плотность вероятности щума неизвестна, но он может быть описан первым и вторым моментами. Сформированный антенной решеткой пространственно-временной сигнал описан в виде линейной модели (7), которая включает и шумовую составляющую. В рамках данной модели (10) представляет собой вектор параметров, который несет информацию о направлении и который необходимо предварительно оценить.

Требования несмещенности и минимума дисперсии оценки реализованы на основе теоремы Гаусса-Маркова в виде матрицы весовых коэффициентов, которыми взвешиваются сигналы на выходе отдельных гидрофонов, в результате чего «отбеливается» шумовая составляющая пространственно-временного сигнала и минимизируется ее дисперсия.

Оценка (13) с помощью матрицы (26) легко преобразуется в дискретную экспоненту (28), у которой пространственная частота определяется направлением на источник отражения/излучения. Тем самым задача оценки направления свелась к задаче оценки частоты гармонического сигнала в «белом» шуме. Анализ решения такой задачи методом максимального правдоподобия показал, что наиболее удобным и реализуемым является метод на основе периодограммы (29), (30).

Необходимо также отметить важное свойство оценки частоты гармонического сигнала: из неравенства Рао-Крамера следует, что дисперсия такой оценки обратно пропорциональна третьей степени числа гидрофонов и обратно пропорциональна отношению сигнал/шум (31). Поэтому изложенная в настоящей статье технология основана на линейной модели представления пространственно-временного сигнала, удовлетворяющей условиям несмещенности и минимума дисперсии оценок, включающей периодограмму для оценки частоты.

Данная технология легко реализуема в современных гидроакустических системах дальнего обнаружения морских объектов и физических явлений и, следовательно, привлекательна для практического применения.

\section{Список литературы}

[1] Монзинго Р.А., Миллер Р.А. Адаптивные антенные решетки. М.: Радио и связь, 1986. 448c. [Monzingo R.A., Miller R.A. Adaptive antenna arrays. Moscow, Radio and Communications, 1986 448p. (in Russian)].

[2] Kay S. Fundamentals of Statistical Signal Processing: Estimation Theory. Prentice-Hall, Englewood Cliffs, N.J., 1993. 595 p.

[3] Haykin S. Adaptive Filter Theory. Prentice-Hall, Englewood Cliffs, N.J., 1986. 590 p.

[4] Hayes M.H. Statistical Digital Signal Processing and Modeling. New York, JOHN WILEY \& SONS, INC., 1996. $622 \mathrm{p}$. 
[5] Марпл С.Л. (мл.) Цифровой спектральный анализ и его приложения. М.: Мир, 1996. 584c. [Marpl S.L. (jun.) Adaptive antenna arrays. Moscow, Mir, 1996. 584p. (in Russian)].

[6] Kay S. Modern Spectral Estimation. Theory and Application. Prentice-Hall, Englewood Cliffs, N.J. 1988. 543 p. 